\title{
THE DENSITY OF INTEGERS REPRESENTABLE AS THE SUM OF FOUR PRIME CUBES
}

\author{
CHRISTIAN ELSHOLTZ AND JAN-CHRISTOPH SCHLAGE-PUCHTA
}

\begin{abstract}
The set of integers which can be written as the sum of four prime cubes has lower density at least 0.009664. This improves earlier bounds of 0.003125 by Ren and 0.005776 by Liu.
\end{abstract}

\section{INTRODUCTION AND RESULTS}

The main result of this article is the following.

Theorem 1. The set of integers which can be written as the sum of four prime cubes has lower density $\omega \geq 0.009664$.

The same result had been obtained by Ren $[3]$ with a density of 0.003125 , which had been improved by Liu [2] to 0.005776 . Note that apart from a set of density 0 , an integer, which is representable as the sum of four prime cubes satisfies the congruence conditions $2 \mid n, n \not \equiv \pm 1, \pm 3(\bmod 9), n \not \equiv \pm 1(\bmod 7)$, thus the density of all integers of this form cannot be larger than $\frac{25}{126}=0.1984 \ldots$.

The proof follows essentially the work of Ren [3], with considerably more precise estimates of an eight-dimensional integral and the singular series. Define $U=(N /(16+\delta))^{1 / 3}, V=U^{5 / 6}, L=\log N$, and denote by $r(n)$ the number of representations of $n$ as $n=p_{1}^{3}+p_{2}^{3}+p_{3}^{3}+p_{4}^{3}$, where $U \leq p_{1}, p_{2} \leq 2 U, V \leq p_{3}, p_{4} \leq 2 V$. (Note that the choice of the exponent $5 / 6$ goes back to Vaughan [5].) Observe that

$$
\frac{1+\varepsilon_{1}(\delta)}{8} N \leq n \leq\left(1+\varepsilon_{2}(\delta)\right) N,
$$

where $\lim _{\delta \rightarrow 0} \varepsilon_{i}(\delta)=0$. Let $I(\delta, N)=\left[\frac{1+\varepsilon_{1}(\delta)}{8} N,\left(1+\varepsilon_{2}(\delta)\right) N\right]$. For very small $\delta$ one can approximately think of $n \in\left[\frac{N}{8}, N\right]$. Then the following holds:

Proposition 2. We have

$$
\sum_{n \in I(\delta, N)} r^{2}(n) \leq(C+o(1)) U V^{4} L^{-8}
$$

where $C=100552$, for sufficiently small $\delta$.

Theorem 1 follows from Proposition 2 by a simple application of Cauchy's inequality. Proposition 2 can also be used to give estimates for integrals occurring in applications of the circle method. We do not go into details here, but refer the reader to the paper [4] by Liu and Lü.

We have

$$
\begin{aligned}
& \sum_{n \in I(\delta, N)} r^{2}(n)= \#\left\{p_{1}^{3}+p_{2}^{3}+p_{3}^{3}+p_{4}^{2}=p_{5}^{3}+p_{6}^{3}+p_{7}^{3}+p_{8}^{3}:\right. \\
&\left.U \leq p_{1}, p_{2}, p_{5}, p_{6} \leq 2 U, V \leq p_{3}, p_{4}, p_{7}, p_{8} \leq 2 V\right\} . \\
&= \#\left\{p_{2}, p_{5}, p_{6} \in[U, 2 U], p_{3}, p_{4}, p_{7}, p_{8} \in[V, 2 V]:\right. \\
&\left.p_{5}^{3}+p_{6}^{3}+p_{7}^{3}+p_{8}^{3}-p_{2}^{3}-p_{5}^{3}-p_{6}^{3} \text { is the cube of a prime }\right\}
\end{aligned}
$$

To bound the quantity on the right we use an upper bound sieve, that is, we first consider the set $\mathcal{M}$ of all integers $m$, such that $p_{5}^{3}+p_{6}^{3}+p_{7}^{3}+p_{8}^{3}-p_{2}^{3}-p_{5}^{3}-p_{6}^{3}=m^{3}$, 
and then we sift out the possible prime values. To do so we need information on the distribution of the elements of $\mathcal{M}$ in residue classes.

Define $R(m)$ as the number of solutions of the equation $m^{3}+p_{2}^{3}+p_{3}^{3}+p_{4}^{3}=$ $p_{5}^{3}+p_{6}^{3}+p_{7}^{3}+p_{8}^{3}$ with $U \leq p_{2}, p_{5}, p_{6} \leq 2 U, V \leq p_{3}, p_{4}, p_{7}, p_{8} \leq 2 \mathrm{~V}$.

We would expect that $R(m)$ is asymptotically equal to a local factor given by some singular series multiplied by a global factor given by an integral involving the density $\frac{1}{\log t}$ of the prime numbers. Unfortunately, we cannot prove this. In fact, we cannot even prove an asymptotic formula for the number of representations of an integer as the sum of 7 cubes. However, if we average over residue classes, then we can prove such a result. This is sufficient, as a linear sieve only requires information on the mean values $\sum_{\substack{U \leq m \leq 2 U \\ d \mid m}} R(m)$. The archimedian part of the asymptotic formula for this sum is given by the integral

$$
J=\frac{1}{3} \int_{\mathfrak{D}} \frac{d v_{1} d v_{2} d v_{3} d v_{4} d u_{1} d u_{2} d u_{3} d u_{4}}{v_{1}^{2 / 3} \cdots v_{4}^{2 / 3} u_{1}^{2 / 3} \cdots u_{4}^{2 / 3} \log v_{1} \cdots \log v_{4} \log u_{1} \cdots \log u_{4}}
$$

where

$$
\begin{aligned}
& \mathfrak{D}=\left\{\left(v_{1}, \ldots, v_{4}, u_{1}, \ldots, u_{4}\right): V^{3} \leq v_{1}, \ldots, v_{4} \leq 8 V^{3}, U^{3} \leq u_{1}, \ldots, u_{4} \leq 8 U^{3},\right. \\
& \left.u_{1}+u_{2}+v_{1}+v_{2}=u_{3}+u_{4}+v_{3}+v_{4}\right\},
\end{aligned}
$$

while the singular series is

$$
\mathfrak{S}_{d}=\sum_{q=1}^{\infty} T_{d}(q)
$$

where

$$
T_{d}(q)=\sum_{(a, q)=1} \frac{S\left(q, a d^{3}\right) C(q, a)^{3} \overline{C(q, a)^{4}}}{q \varphi(q)^{7}}
$$

and

$$
S(q, a)=\sum_{m=1}^{q} e\left(\frac{a m^{3}}{q}\right), \quad C(q, a)=\sum_{(m, q)=1} e\left(\frac{a m^{3}}{q}\right) .
$$

To summarize these considerations: the estimation of $\sum r^{2}(n)$ falls into four tasks:

(1) estimate the distribution of $R$ on residue classes

(2) estimate $J$

(3) estimate $\mathfrak{S}_{d}$

(4) apply an upper bound sieve. For this last step we use the weighted version of Iwaniecs's sieve due to Kawada and Wooley.

\section{STEP 1}

For the first part we use results by Ren [3] and Liu [2] as follows.

Define $E_{d}$ by means of the equation

$$
\sum_{\substack{U \leq m \leq 2 U \\ d \mid m}} R(m)=\frac{\mathfrak{S}_{d}}{d} J+E_{d} .
$$

Then we have the following:

Lemma 3. (1) $\mathfrak{S}_{d}$ is absolutely convergent and bounded independently of $d$. 
(2) Let $\epsilon>0$ be fixed and let $\tau$ denote the divisor function. For all complex numbers $\eta_{d}$ satisfying $\left|\eta_{d}\right| \leq \tau(d)$ and for all fixed $A$ the following estimate holds:

$$
\sum_{d \leq N^{11 / 90-\epsilon}} \eta_{d} E_{d} \ll U V^{4} L^{-A}
$$

The first part of the statement is contained in Ren's result [3, Lemma 3.2], the second statement is due to Liu [2, Lemma 3.2].

\section{STEP 2}

We now compute $J$.

Lemma 4. We have $J \leq 440.62 U V^{4} L^{-7}$.

Proof. As each of the variables $u_{1}, u_{2}, u_{3}, u_{4}, v_{1}, v_{2}, v_{3}, v_{4}$ varies over its range of integration in $\mathfrak{D}$, it changes by a factor 8 at most. Hence its logarithm changes by $\mathcal{O}(1)$, and therefore

$$
\log v_{i}=\left(1+\mathcal{O}\left(\frac{1}{\log V}\right)\right) \log V^{3}=(1+o(1)) \frac{5}{6} L
$$

and

$$
\log u_{i}=\left(1+\mathcal{O}\left(\frac{1}{\log U}\right)\right) \log U^{3}=(1+o(1)) L
$$

We conclude that

$J=\frac{(1+o(1)) 6^{4}}{3 \cdot 5^{4} L^{8}} \int_{V^{3}}^{8 V^{3}} \frac{d v_{1}}{v_{1}^{2 / 3}} \cdots \int_{V^{3}}^{8 V^{3}} \frac{d v_{4}}{v_{4}^{2 / 3}} \int_{U^{3}}^{8 U^{3}} \frac{d u_{1}}{u_{1}^{2 / 3}} \int_{U^{3}}^{8 U^{3}} \frac{d u_{2}}{u_{2}^{2 / 3}} \int_{\max \left(U^{3}, x-8 U^{3}\right)}^{\min \left(8 U^{3}, x-U^{3}\right)} \frac{d u_{3}}{u_{3}^{2 / 3}\left(x-u_{3}\right)^{2 / 3}}$,

where $x-u_{3}=v_{1}+v_{2}-v_{3}-v_{4}+u_{1}+u_{2}-u_{3}=u_{4}$. If we replace $x$ by $x^{\prime}=$ $x-\left(v_{1}+v_{2}-v_{3}-v_{4}\right)=u_{1}+u_{2}-u_{3}$, the range of the innermost integral changes by $\mathcal{O}\left(V^{3}\right)$. Since the integrand is $\mathcal{O}\left(V^{-8} U^{-8}\right)$, and the range of the first six integrals is $\mathcal{O}\left(V^{12} U^{6}\right)$, this change in the range introduces an error $\mathcal{O}\left(V^{7} U^{-2}\right)=\mathcal{O}\left(U^{23 / 6}\right)$, which is of smaller order of magnitude than $U V^{4} L^{-7} \gg\left(U^{13 / 3-\varepsilon}\right)$. The change of the innermost integrand is

$$
\ll \frac{\left|x-x^{\prime}\right|}{u_{3}^{2 / 3}\left(x-u_{3}\right)^{5 / 3}} \ll \frac{\left|x-x^{\prime}\right|}{U^{7}} \ll V^{3} U^{-7}
$$

The total range of integration has measure $\ll V^{12} U^{9}$, and the factors outside the innermost integral are $\mathcal{O}\left(V^{-8} U^{-4}\right)$, hence this change introduces an error of 
magnitude $\mathcal{O}\left(V^{7} U^{-2}\right)=\mathcal{O}\left(U^{23 / 6}\right)$, which is also negligible. Hence

$$
\begin{aligned}
J & =\frac{(1+o(1)) 6^{4}}{3 \cdot 5^{4} L^{7}} \int_{V^{3}}^{8 V^{3}} \frac{d v_{1}}{v_{1}^{2 / 3}} \cdots \int_{V^{3}}^{8 V^{3}} \frac{d v_{1}}{v_{4}^{2 / 3}} \int_{U^{3}}^{8 U^{3}} \frac{d u_{1}}{u_{1}^{2 / 3}} \int_{U^{3}}^{8 U^{3}} \frac{d u_{2}}{u_{2}^{2 / 3}} \\
\min \left(8 U^{3}, u_{1}+u_{2}-U^{3}\right) & \int_{\max \left(U^{3}, u_{1}+u_{2}-8 U^{3}\right)}^{u_{3}^{2 / 3}\left(u_{1}+u_{2}-u_{3}\right)^{2 / 3}} \\
& =\frac{18^{4}+o(1)}{3 \cdot 5^{4} L^{7}} V^{4} \int_{U^{3}}^{8 U^{3}} \frac{d u_{1}}{u_{1}^{2 / 3}} \int_{U^{3}}^{8 U^{3}} \frac{d u_{2}}{u_{2}^{2 / 3}} \int_{\max \left(U^{3}, u_{1}+u_{2}-8 U^{3}\right)}^{\min \left(8 \cdot U^{3}, u_{1}+u_{2}-U^{3}\right)} \frac{d u_{3}}{u_{3}^{2 / 3}\left(n+u_{1}+u_{2}-u_{3}\right)^{2 / 3}} \\
& =\frac{18^{4}+o(1)}{3 \cdot 5^{4} L^{7}} U V^{4} \int_{1}^{8} \frac{d t_{1}}{t_{1}^{2 / 3}} \int_{1}^{8} \frac{d t_{2}}{t_{2}^{2 / 3}} \int_{\max \left(1, t_{1}+t_{2}-8\right)}^{t_{3}^{2 / 3}\left(t_{1}+t_{2}-t_{3}\right)^{2 / 3}} .
\end{aligned}
$$

The triple integral can be computed numerically to be in the range $[7.85,7.87]$. We conclude that

$$
J \leq(1+o(1)) \frac{18^{4}}{3 \cdot 5^{4}} 7.87 \frac{U V^{4}}{L^{7}}<440.62 \frac{U V^{4}}{L^{7}},
$$

provided that $N$ is sufficiently large.

The reader might wonder why we did not integrate numerically from the start, however, giving a provable bound for an eight dimensional integral is quite a delicate task.

\section{STEPS 3 AND 4}

We now apply the weighted version of Iwaniec's sieve due to Kawada and Wooley (see [1, Lemma 9.1]). We do not want to go into details here, as the computations are the same as in the work of Ren [3, Lemma 4.17]. We obtain

$$
\sum_{n \in I(\delta, N)} r^{2}(n) \leq(1+o(1)) e^{\gamma} J \mathfrak{S}_{1} W(z)
$$

where $\gamma=0.577 \ldots$ is Euler's constant, and

$$
\begin{aligned}
W(z) & =\prod_{p \leq N^{11 / 180-\epsilon}}\left(1-\frac{\omega(p)}{p}\right), \\
\mathfrak{S}_{1} & =\left(1+T_{1}(3)+T_{1}(9)\right) \prod_{p \neq 3}\left(1+T_{1}(p)\right), \\
\omega(p) & =\frac{1+T_{p}(p)}{1+T_{1}(p)} .
\end{aligned}
$$

The singular series $\mathfrak{S}_{d}$ mentioned in the introduction enters into the sieve, its contribution can be seen in the structure of the product $W(z)$. We have

$$
1-\frac{\omega(p)}{p}=\left(1-\frac{1}{p}\right)\left(1-\frac{T_{p}(p)-T_{1}(p)}{p-1}\right) .
$$


Using Mertens' estimate $\prod_{p<z}\left(1-\frac{1}{p}\right) \sim e^{-\gamma} \frac{1}{\log z}$ and the fact that $\prod_{p \geq 11}\left(1-\frac{T_{p}(p)-T_{1}(p)}{p-1}\right)$ converges we obtain for $z=N^{11 / 180-\epsilon}$

$$
\begin{aligned}
\prod_{11 \leq p \leq N^{11 / 180-\epsilon}}\left(1-\frac{\omega(p)}{p}\right) & =(1+o(1)) \frac{e^{-\gamma}}{\log z} \cdot 2 \cdot \frac{3}{2} \frac{5}{4} \frac{7}{6} \prod_{p \geq 11}\left(1-\frac{T_{p}(p)-T_{1}(p)}{p-1}\right) \\
& \leq \frac{40.197}{\log N} \prod_{p \geq 11}\left(1-\frac{T_{p}(p)-T_{1}(p)}{p-1}\right)
\end{aligned}
$$

provided that $\epsilon$ is sufficiently small.

If $p \mid a$, then $S(p, a)=p$. If $p \nmid a$, then we have by Weil-estimates $|S(p, a)| \leq 2 \sqrt{p}$, and therefore $|C(p, a)| \leq 2 \sqrt{p}+1$. Hence

$$
\left|T_{p}(p)\right| \leq\left(\frac{2 \sqrt{p}+1}{p-1}\right)^{7}, \quad\left|T_{1}(p)\right| \leq \frac{2}{\sqrt{p}}\left(\frac{2 \sqrt{p}+1}{p-1}\right)^{7},
$$

and we see that the product over $p$ converges so fast that it can easily be evaluated numerically.

More precisely we have

$$
\begin{aligned}
\prod_{p \geq P}\left(1-\frac{T_{p}(p)-T_{1}(p)}{p-1}\right) & \leq \prod_{p \geq P}\left(1+2 \frac{(\sqrt{p}+2)(2 \sqrt{p}+1)^{7}}{\sqrt{p}(p-1)^{7}}\right) \\
\leq & \prod_{p \geq P}\left(1+\frac{M}{p^{7 / 2}}\right) \leq\left(\frac{\zeta(7 / 2)}{\zeta(7)} \prod_{p<P}\left(1+\frac{1}{p^{7 / 2}}\right)^{-1}\right)^{M},
\end{aligned}
$$

where $M=2\left(1-\frac{1}{P}\right)^{-7}\left(1+\frac{2}{\sqrt{P}}\right)\left(2+\frac{1}{\sqrt{P}}\right)^{7}$.

Using $P=4000$ we obtain $M=279.551$, and write

$$
\begin{aligned}
\prod_{p \geq 11}\left(1-\frac{T_{p}(p)-T_{1}(p)}{p-1}\right) \leq & \prod_{11 \leq p \leq 200}\left(1-\frac{T_{p}(p)-T_{1}(p)}{p-1}\right) \prod_{200 \leq p \leq 4000}\left(1+2 \frac{(\sqrt{p}+2)(2 \sqrt{p}+1)^{7}}{\sqrt{p}(p-1)^{7}}\right) \\
& \quad\left(\frac{\zeta(7 / 2)}{\zeta(7)} \prod_{p<4000}\left(1+\frac{1}{p^{7 / 2}}\right)^{-1}\right)^{M} \\
\leq & 1.029437\left(1+1.92 \cdot 10^{-5}\right)\left(1+4.54 \cdot 10^{-11}\right) \leq 1.02944 .
\end{aligned}
$$

Plugging this value into the estimate above we obtain

$$
\prod_{11 \leq p \leq N^{11 / 180-\epsilon}}\left(1-\frac{\omega(p)}{p}\right) \leq \frac{41.38}{\log N} .
$$

Similarly we can estimate $\mathfrak{S}_{1}$ as

$$
\begin{aligned}
& \mathfrak{S}_{1} \leq\left(1+T_{1}(3)+T_{1}(9)\right) \prod_{5 \leq p \leq 500}\left(1+T_{1}(p)\right) \prod_{500 \leq p \leq 4000}\left(1+\frac{2(2 \sqrt{p}+1)^{7}}{\sqrt{p}(p-1)^{7}}\right) \\
&\left(\frac{\zeta(4)}{\zeta(8)} \prod_{p<4000}\left(1+\frac{1}{p^{4}}\right)^{-1}\right)^{270.982} \\
& \leq 3.0963\left(1+1.19 \cdot 10^{-7}\right)\left(1+1.64 \cdot 10^{-10}\right) \leq 3.0964 .
\end{aligned}
$$




\section{Putting these Results together}

Putting these results together we obtain

$\sum_{n \in I(\delta, N)} r^{2}(n) \leq(1+o(1)) e^{\gamma} 440.62 \cdot 41.3794 \cdot 3.0964 \cdot U V^{4} L^{-8} \leq 100552 U V^{4} L^{-8}$ and the proof of Proposition 2 is complete.

To deduce Theorem 1 we apply the Cauchy-Schwarz inequality in much the same way as in the proof of Romanov's theorem. We have

$$
\sum_{n \in I(\delta, N)} r(n)=(\pi(2 U)-\pi(U))^{2}(\pi(2 V)-\pi(V))^{2} \sim \frac{3^{4} \cdot 6^{2}}{5^{2}} U^{2} V^{2} L^{-4},
$$

thus

$$
\begin{aligned}
\sum_{\substack{n \in I(\delta, N) \\
r(n) \geq 1}} 1 \geq\left(\sum_{n \in I(\delta, N)} r(n)\right)^{2}\left(\sum_{n \in I(\delta, N)} r^{2}(n)\right)^{-1} \\
\quad \geq \frac{3^{8} \cdot 6^{4}}{5^{4} \cdot 100552} U^{3}=(1+O(\delta)) \frac{3^{12}}{5^{4} \cdot 100552} N \geq 0.00845638 N,
\end{aligned}
$$

for sufficiently small $\delta$. Patching intervals of the form $[N / 8, N]$ together Theorem 1 follows by multiplying the last density with $8 / 7$.

The authors would like to thank the referee for useful comments on the manuscript.

\section{REFERENCES}

[1] K. Kawada, T. Wooley, On the Waring-Goldbach problem for fourth and fifth powers, Proc. London Math. Soc. 83 (2001), 1-50.

[2] Z. Liu, Density of the sums of four cubes of primes, J Number Theory 132 (2012), 735-747.

[3] X. Ren, Sums of four cubes of primes, J. Number theory 98 (2003), 156-171.

[4] Z. Liu, G. Lü, Eight cubes of primes and powers of 2, Acta Arith. 145 (2010), 171-192.

[5] R.C. Vaughan, Sums of three cubes. Bull. London Math. Soc. 17 (1985), 17-20.

\section{Key words and MSC:}

Goldbach-Waring problem, applications of sieve methods, sums of cubes

Primary: 11P32 Goldbach-type theorems; other additive questions involving primes Secondary: 11P05 Waring's problem and variants

11N36 Applications of sieve methods

Christian Elsholtz, Institut für Analysis und Zahlentheorie, Technische Universität Graz, A-8010 Graz, Austria

Jan-Christoph Schlage-Puchta, Mathematical institute, University Rostock, 18957 Rostock, Germany 